\title{
Pure Absorption-Mode Spectra Using a Modulated RF Mixing Period in MQMAS Experiments
}

\author{
Thomas Vosegaard,* Pierre Florian,* Philip J. Grandinetti, $\dagger$ and Dominique Massiot* \\ *CRMHT-CNRS, $1 D$ avenue de la Recherche Scientifique, 45071 Orléans Cedex 2, France; and \\ $\dagger$ Department of Chemistry, Ohio State University, Columbus, Ohio \\ Received July 30, 1999; revised October 8, 1999
}

\begin{abstract}
Different approaches to obtain pure absorption-mode lineshapes in MQMAS experiments employing a train of $180^{\circ}$ phasealternating pulses for the multiple-quantum to single-quantum mixing period are investigated. Four pulse sequences, which achieve this by using either the shifted-echo approach or the hypercomplex approach with symmetric coherence transfer pathways, are presented and their improved lineshape- and sensitivityperformance is experimentally demonstrated by ${ }^{87} \mathrm{Rb}$ MQMAS of $\mathrm{RbNO}_{3}$. Compared to the original modulated-rf mixing sequence, sensitivity enhancements by factors up to 1.3 are obtained with the sequences described here. ๑ 2000 Academic Press
\end{abstract}

Key Words: MQMAS; modulated RF pulses; pure absorptionmode spectra.

The field of high-resolution solid-state NMR of half-integer quadrupolar nuclei has experienced several important advances during the past decade. One of the most recent milestones within this field was the introduction of the multiple-quantum magic-angle spinning (MQMAS) experiment by Frydman and Harwood (1). Like the double rotation $(2,3)$ and dynamicangle spinning (3-5) techniques the MQMAS experiment provides isotropic spectra of half-integer quadrupolar nuclei dominated by the second-order quadrupolar broadening which is only partly averaged by MAS. As this experiment only uses MAS and combined multiple-quantum and single-quantum coherence evolution periods to achieve this, it enjoys the benefit that its implementation requires only a simple MAS probe. However, the critical factor of the MQMAS experiment is that it relies on efficient excitation and reconversion of the multiple-quantum coherences. Unfortunately, these are normally associated with low transfer amplitudes, which furthermore decrease with increasing quadrupole couplings. Therefore, the applicability of the MQMAS experiment is often limited by a low sensitivity, especially for nuclei with large quadrupole couplings. While numerous groups have proposed alternative schemes for improved multiple-quantum excitation $(6-18)$, less attention has been drawn to optimizing the multiple-quantum to single-quantum mixing $(7,14,18)$.

Based on the early work of Vega and Naor (19) some very recent studies have demonstrated that significant sensitivity enhancements of the MQMAS experiment for spin $I=3 / 2$ nuclei can be obtained by optimizing the triple- to singlequantum reconversion (20-22), i.e., using modulated pulses that selectively irradiate the satellite transitions. In addition to an improved mixing efficiency they also found a highly selective excitation of the $p=0 \rightarrow-3 \rightarrow-1$ coherence pathway over the $p=0 \rightarrow+3 \rightarrow-1$ pathway (22). This selectivity has the drawback that it inhibits the acquisition of the $p=0 \rightarrow$ $+3 \rightarrow-1$ pathway signal which is necessary in the hypercomplex or TPPI approaches to pure absorption-mode lineshapes.

In this Communication we present four MQMAS sequences with modulated pulses that provide pure absorption-mode 2D spectra while retaining the sensitivity gain obtained with modulated-RF mixing period. The pure absorption-mode lineshapes are achieved by using either the shifted-echo approach $(9,14,23,24)$ or the hypercomplex approach $(25)$ with symmetric coherence transfer pathways $(26,27)$.

The MQMAS pulse sequence with modulated-RF mixing period proposed by Madhu et al. (2l) is shown in Fig. 1a. We will use this sequence as the basis for our modified acquisition schemes because it is easier to implement than the double frequency-sweep sequence presented by Kentgens and Verhagen (20). It should be noted that although these two sequences rely on similar physical principles they are still quite different because the latter sequence sweeps the carrier frequency over the entire satellite spectrum (20). On the other hand, the modulated pulses used by Madhu et al. (21) correspond to irradiation at two distinct frequencies, ideally on the singularities of the satellite powder pattern. This pulse sequence consists of a triple-quantum excitation pulse followed by the triple-quantum evolution period $t_{1}$. The triple-quantum to single-quantum reconversion is performed by the $\left(p_{x}(\tau)-\tau-p_{\bar{x}}(\tau)-\tau\right)_{n}$ block of pulses which represents a simple but easy-to-implement approximation to a cosine modulation of the RF signal. The acquisition begins immediately after the reconversion pulses. Figure $1 \mathrm{~b}$ shows the ${ }^{87} \mathrm{Rb}$ free-induction decay (FID) for a sample of $\mathrm{RbNO}_{3}$ obtained by this pulse sequence and split into the $p=0 \rightarrow-3 \rightarrow-1$ and $p=0 \rightarrow+3 \rightarrow-1$ signals. We note that the $p=0 \rightarrow+3 \rightarrow-1$ signal is almost 
a
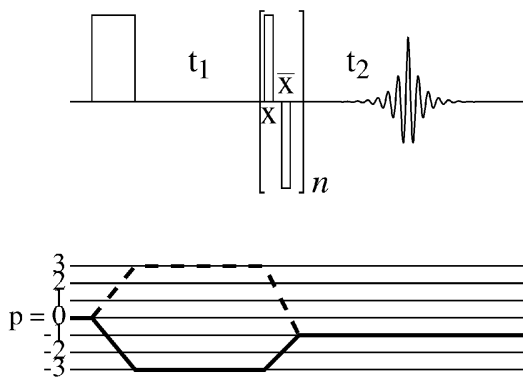

b

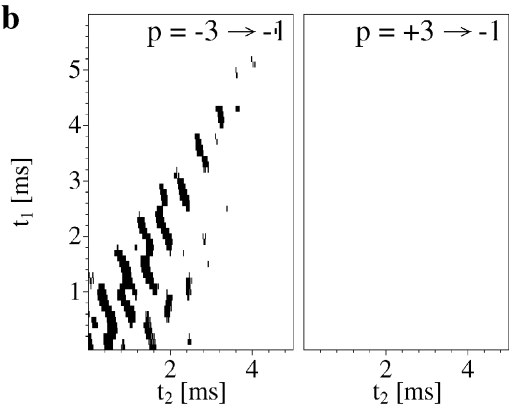

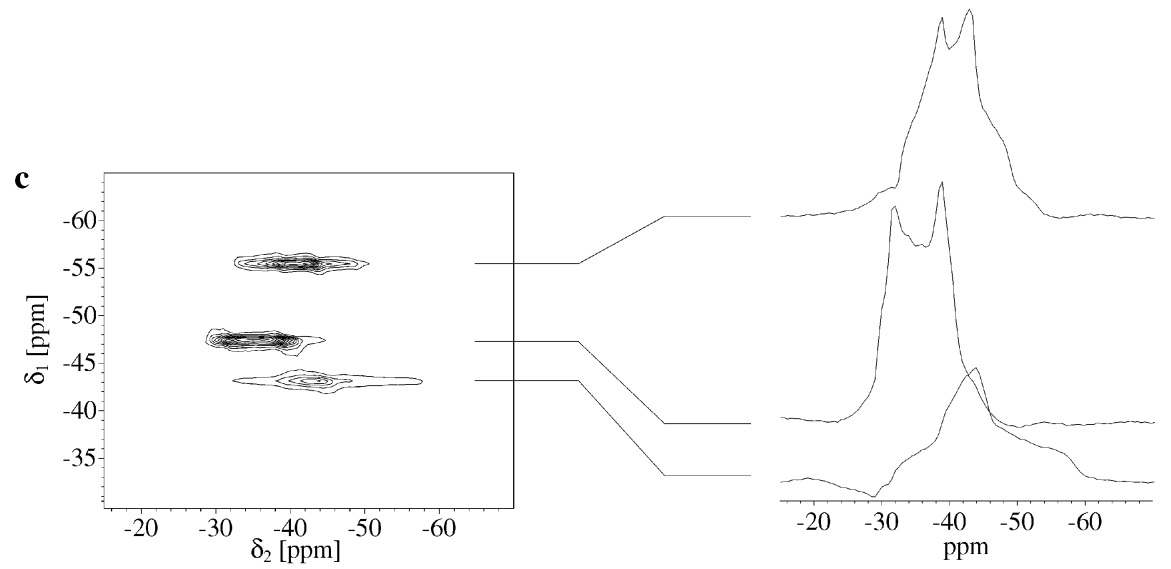

FIG. 1. (a) MQMAS pulse sequence with modulated triple- to single-quantum conversion pulses as proposed by Madhu et al. (21). (b) ${ }^{87} \mathrm{Rb}$ FID of RbNO ${ }_{3}$ obtained by this pulse sequence recorded on a Bruker DSX 300 spectrometer $\left(7.0 \mathrm{~T}\right.$ ) with a larmor frequency for ${ }^{87} \mathrm{Rb}$ of $98.2 \mathrm{MHz}$. The spectrum was recorded employing $10 \mathrm{kHz}$ MAS, a spectral width of $50 \mathrm{kHz}$, rotor synchronized $t_{1}$ incrementation (100 $\left.\mu \mathrm{s}\right)(28)$, RF field strengths of $100 \mathrm{kHz}$ for the triple-quantum excitation pulse $\left(\tau_{\text {pulse }}=4 \mu \mathrm{s}\right)$ and $80 \mathrm{kHz}$ for the triple- to single-quantum reconversion pulses $\left(\tau_{\text {pulse }}=\tau_{\text {delay }}=1 \mu \mathrm{s}\right)$ with an optimized number of loops $(n=$ 3 ), and 48 transients followed by a repetition delay of $2 \mathrm{~s}$ for each $t_{1}$ slice. (c) Isotropic/anisotropic spectrum obtained by a shearing transformation (6, 9, 11$)$ of the FID in (b). This spectrum employs $50 \mathrm{~Hz}$ shifted-gaussian apodization in the $t_{2}$ dimension. Contours are drawn at $10,20, \ldots, 90 \%$ of the maximum intensity.

absent in accordance with the fact that the modulated pulses mainly transfer the $p=0 \rightarrow-3 \rightarrow-1$ signal.

The selectivity obtained by the cosine-modulated mixing pulses may easily be justified by looking at the effective Hamiltonian for a spin-3/2 nucleus during the mixing pulse

$$
\begin{aligned}
\mathscr{H}_{\mathrm{eff}}= & \omega_{\mathrm{Q}}(\theta, \phi)\left(I_{z}^{1-2}-I_{z}^{3-4}\right) \\
& -\omega_{1}(t)\left(\sqrt{3}\left(I_{x}^{1-2}+I_{x}^{3-4}\right)+2 I_{x}^{2-3}\right),
\end{aligned}
$$

where the quadrupolar frequency $\left(\omega_{\mathrm{Q}}\right)$ is given by $\omega_{\mathrm{Q}}(\theta, \phi)=$ $e^{2} q Q / 2 \hbar \times\left(1 / 2\left(3 \cos ^{2} \theta-1\right)+\eta \sin ^{2} \theta \cos 2 \phi\right), \omega_{1}(t)=$ $\omega_{1} \cos \omega_{m} t$, and $I_{\alpha}^{i-j}$ represent fictitious spin-1/2 operators (29). For our purposes it is convenient to express this Hamiltonian in a frame that rotates about the quadrupolar interaction $\left(I_{z}^{1-2}-\right.$ $I_{z}^{3-4}$ ) with an angular frequency of $\omega_{m}$, as proposed by Vega and Naor (19). In this frame, which does not influence the triple-quantum- and central-transition single-quantum-operators, the effective Hamiltonian takes the form

$$
\begin{aligned}
\tilde{\mathscr{H}}_{\mathrm{eff}}= & {\left[\omega_{\mathrm{Q}}(\theta, \phi)-\omega_{m}\right]\left(I_{z}^{1-2}-I_{z}^{3-4}\right) } \\
& -2 \omega_{1} \cos \left(\omega_{m} t\right) I_{x}^{2-3}-\frac{\sqrt{3}}{2} \omega_{1}\left[\left(I_{x}^{1-2}+I_{x}^{3-4}\right)\right. \\
& \left.\times\left(1+\cos 2 \omega_{m} t\right)-\left(I_{y}^{1-2}+I_{y}^{3-4}\right) \sin 2 \omega_{m} t\right] .
\end{aligned}
$$

Neglecting all the time-dependent terms, which will average to zero over a period of $2 \pi / \omega_{m}$, and assuming that it is possible to choose $\omega_{m}$ to be on resonance with $\omega_{\mathrm{Q}}(\theta, \phi)$ for elimination of the first term, the effective Hamiltonian reduces to

$$
\tilde{\mathscr{H}}_{\mathrm{eff}} \approx-\frac{\sqrt{3}}{2} \omega_{1}\left(I_{x}^{1-2}+I_{x}^{3-4}\right) .
$$

Immediately before the mixing pulses, described by the effective Hamiltonian above, we assume that the spin system contains only triple-quantum coherence, represented by, e.g., $I^{1-4}$ ( $p=-3$ coherence). Suppose the mixing period is of duration 
a

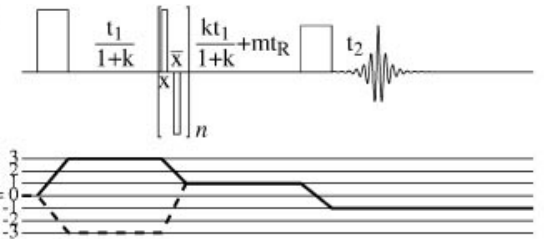

b
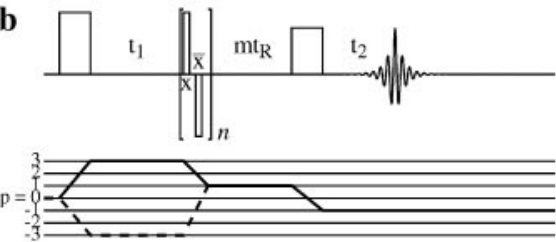

c
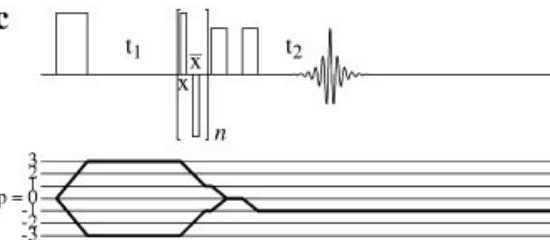

d
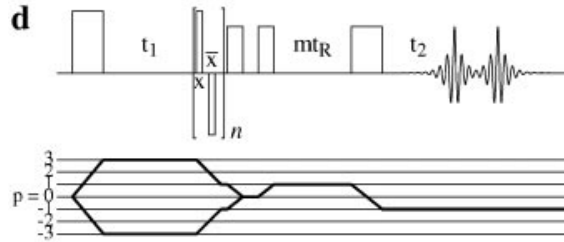
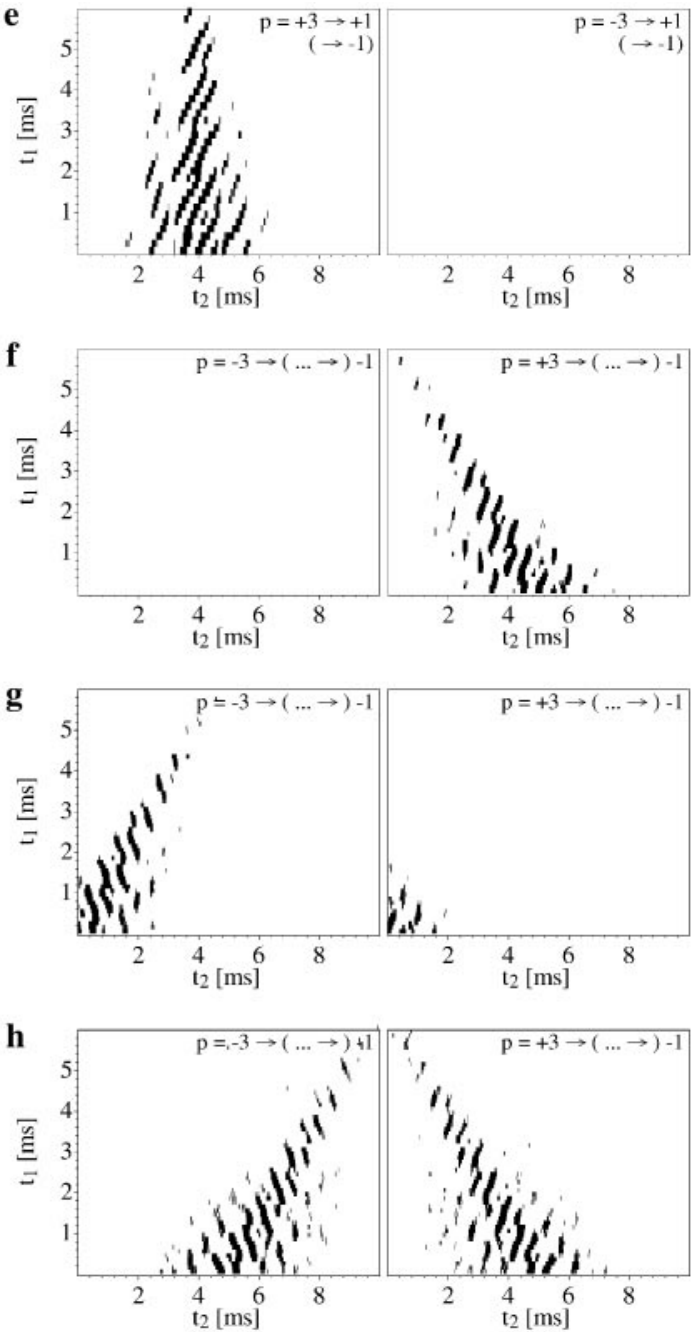

FIG. 2. (a-d) MQMAS pulse sequences with modulated-RF mixing period yielding pure absorption-mode spectra by (a,b) the split- $t_{1}$ and shifted-echo approaches where the echo is shifted with a central-transition selective $\pi$ pulse, (c) hypercomplex acquisition of symmetric pathways (the duration of the selective pulse immediately after the modulated pulses is tuned to optimize the signal and may vary slightly from a $\pi / 2$ pulse), or (e) a combination of the shifted-echo and hypercomplex sequences. For the four sequences 24 or 48 step phase cyclings were applied to select the indicated pathways. (e-h) ${ }^{87} \mathrm{Rb}$ free-induction decays for a sample of $\mathrm{RbNO}_{3}$ for the two-dimensional experiments in (a-d) and obtained under the same conditions as the FID in Fig. $1 \mathrm{~b}$ and split into the $p=0 \rightarrow$ $\pm 3 \rightarrow-1$ signals. The RF-field strength for the selective pulses was $40 \mathrm{kHz}$.

$\tau_{\text {mix }}=2 \pi /\left(\sqrt{3} \omega_{1}\right)$, the evolution during the mixing period yields

$$
\begin{aligned}
& e^{-i \tilde{\mathscr{H}}_{\mathrm{eff}} \mathrm{T}_{\mathrm{mix}}} I_{-}^{1-4} e^{i \tilde{\mathscr{H}}_{\mathrm{eff}} \tau_{\mathrm{mix}}} \\
& =e^{i \pi\left(I_{x}^{1-2}+I_{x}^{3-4}\right)} I_{-}^{1-4} e^{-i \pi\left(I_{x}^{1-2}+I_{x}^{3-4}\right)}=-I_{-}^{2-3} .
\end{aligned}
$$

From this equation it is clear that under the conditions described above, the cosine-modulated mixing pulses select uniquely the $p=0 \rightarrow-3 \rightarrow-1$ coherence pathway. Moreover, it is also clear why the modulated-RF sequences give improved sensitivity, because the Hamiltonian in Eq. [3] transfers $I^{1-4}$ into $I^{2-3}$ without loss to other coherences. It should be noted that Vega and co-workers (30) are working on a more extensive description of the modulated-RF mixing.

The isotropic/anisotropic correlation spectrum shown in Fig. $1 \mathrm{c}$ is obtained by a shearing transformation of the $p=0 \rightarrow$ $-3 \rightarrow-1$ signal of the FID in Fig. 1. The contour plot clearly reveals three distinct rubidium sites in accordance with the crystal structure (31) and previously published NMR results $(9,11,24,32)$. However, when taking a closer look at the cross sections through the three isotropic peaks we observe significant phase distortions of the lineshapes compared to ideal second-order quadrupolar lineshapes. These distortions show that this sequence does not provide a pure absorption-mode spectrum.

To obtain a pure absorption-mode 2D spectrum while taking 

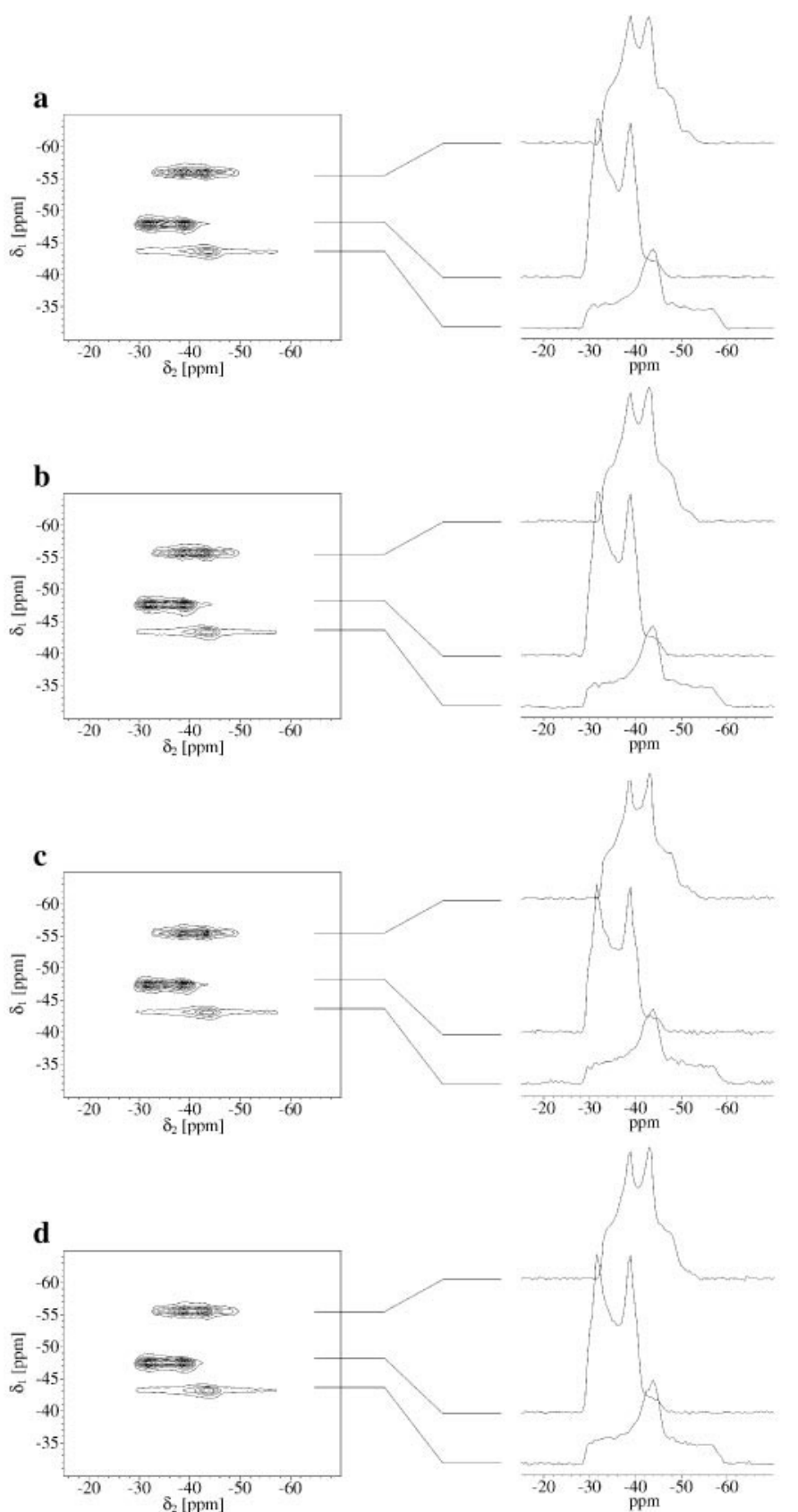

FIG. 3. ${ }^{87} \mathrm{Rb}$ MQMAS spectra of $\mathrm{RbNO}_{3}$ obtained by shearing transformations (except from the FID in Fig. 2e that requires no shearing) of the FIDs in Fig. 2 with the spectrum in (a) corresponding to the FID in Fig. 2e, (b) to Fig. 2f, (c) to Fig. 2g, and (d) to Fig. 2h. For each spectrum the FIDs were weighted with $50 \mathrm{~Hz}$ shifted-gaussian line broadening, processed, and for the spectra in (c) and (d) the spectra for the two pathways were added to yield the spectra shown. Contours are drawn at $10,20, \ldots, 90 \%$ of the maximum intensity.

advantage of the improved sensitivity of the pulse sequence in Fig. 1a, we designed the four pulse sequences shown in Figs. 2a-2d. The simplest sequences (Figs. 2a and 2b) use the shifted-echo approach $(9,24)$ to obtain pure absorption-mode lineshapes. The sequence in Fig. 2a uses a delayed acquisition
(14) in which the $t_{1}$ period is split between evolution periods as $p=3$ and $p=1$. Consequently, the 2D FID, shown in Fig. $2 \mathrm{e}$, contains echo tops that do not advance with increasing $t_{1}$, and because of the selective mixing pulses contains only the $p=0 \rightarrow+3 \rightarrow+1(\rightarrow-1)$ coherence pathway. When there is an inhomogeneous broadening associated with the isotropic dimension (as often occurs in glasses), then the shifted-echo sequence in Fig. $2 b$ may be able to refocus a greater signal volume and therefore would be preferred over the sequence in Fig. 2a. In this experiment the delay between the mixing pulses and the selective $\pi$ pulse is constant. The FID (Fig. 2f) is now dominated by the $p=0 \rightarrow+3(\rightarrow+1) \rightarrow-1$ pathway, and we note that for a crystalline compound the echo shifts toward lower $t_{2}$ values with increasing $t_{1}$. As an alternative to the shifted-echo sequences, acquisition of both of the \pm 3 quantum coherence pathways may also provide a pure absorption-mode spectrum. The sequence in Fig. 2c creates symmetric pathways by use of two central-transition selective $\pi / 2$ pulses immediately after the mixing period (25-27). As the antiecho signal is shifted to negative $t_{2}$ values and therefore only partly detectable, the FID obtained by this hypercomplex sequence (Fig. $2 \mathrm{~g}$ ) is dominated by the echo signal. Finally, to acquire the full $p=0 \rightarrow-3 \rightarrow \ldots \rightarrow-1$ and $p=0 \rightarrow+3 \rightarrow \ldots \rightarrow-1$ signals we have combined the hypercomplex and shifted-echo sequences as shown in Fig. 2d. Employing this sequence the FID (Fig. 2h) indeed contains both pathway signals with same intensity. Finally, it should be emphasized that the selectivity of the modulated mixing pulses may be used to reduce the phase cycling in other MQMAS experiments as they require $p= \pm 3 \rightarrow \pm 1$ coherence pathway selection of the mixing pulse $(14,27,33)$.

Two-dimensional Fourier transforms of the FIDs in Figs. $2 \mathrm{e}-2 \mathrm{~h}$ are shown in Fig. 3. For the FIDs in Figs. $2 \mathrm{~g}$ and $2 \mathrm{~h}$ the spectra are obtained by separate processing of the two pathways which are added to yield the spectra shown. It is noted that the FID from the hypercomplex shifted-echo sequence (Fig. 2d) provides pure absorption-mode spectra from either the $p=0 \rightarrow+3 \rightarrow \ldots \rightarrow-1$ or the $p=0 \rightarrow-3 \rightarrow \ldots \rightarrow$ -1 pathway signals. The contour plots of all four sequences

TABLE 1

Signal to Noise Ratios of the Cross Sections through the Three Sites of the ${ }^{87} \mathrm{Rb}$ MQMAS Spectra of $\mathrm{RbNO}_{3}$

\begin{tabular}{llll}
\hline \multicolumn{1}{c}{ Pulse sequence (Figure) } & Site 1 & Site 2 & Site 3 \\
\hline Original (1a) & 1 & 1 & 1 \\
Split- $t_{1}$ (2a) & 1.27 & 1.32 & 1.21 \\
Shifted echo (2b) & 1.21 & 1.24 & 1.12 \\
Hypercomplex (2c) $_{\text {Hypercomplex shifted echo (2d) }}$ & 0.56 & 0.56 & 0.44 \\
Two-pulse sequence $^{a}$ & 0.77 & 0.85 & 0.74 \\
\hline
\end{tabular}

Note. For all sites the $\mathrm{S} / \mathrm{N}$ ratio is given relative to the $\mathrm{S} / \mathrm{N}$ ratio achieved by the sequence in Fig. 1.

${ }^{a}$ Two-pulse sequence with single-pulse mixing $(6,8,9,11)$. 
resemble the spectrum in Fig. 1c obtained by the original sequence (21). However, looking at the cross sections of the three sites, we observe a significant improvement of the lineshape performance of all four sequences compared to the sequence in Fig. 1. Thus, the proposed sequences all eliminate the dispersion-mode lineshapes of the original experiment proposed by Madhu et al. (2l).

Provided there is no significant damping of the signal due to $T_{2}$ relaxation, which is the case for ${ }^{87} \mathrm{Rb}$ in $\mathrm{RbNO}_{3}$, the sensitivity should increase when the whole echo is acquired (24). To investigate the resulting improvement on the complete $2 \mathrm{D}$ spectra, Table 1 summarizes the $\mathrm{S} / \mathrm{N}$ ratios for the traces of the three sites for the four present sequences relative to the sequence in Fig. 1. Indeed the improved $\mathrm{S} / \mathrm{N}$ ratio obtained by acquiring the whole echo is also reflected in the complete $2 \mathrm{D}$ spectrum. Thereby, sensitivity improvements by factors up to 1.32 are obtained using the shifted-echo sequences. The two hypercomplex sequences (Figs. 2c and 2d) do not provide improved $\mathrm{S} / \mathrm{N}$ ratios, most likely due to the increased number of pulses employed. Moreover, the two hypercomplex sequences suffer the usual decrease in the $\mathrm{S} / \mathrm{N}$ ratio by employing symmetric pathways (24). To evidence the improved sensitivity obtained using modulated-RF mixing, the $\mathrm{S} / \mathrm{N}$ ratios for the simple two-pulse sequence $(6,8,9,11)$ are included in Table 1. Indeed we achieve a significant improvement of the sensitivity employing modulated-RF mixing, i.e., improvements of the $\mathrm{S} / \mathrm{N}$ ratio by factors up to 2.75 are achieved by the shifted-echo sequences relative to the single-pulse mixing sequence (for $\omega_{1} / 2 \pi \approx 80 \mathrm{kHz}$ ).

It is of interest to investigate the possible applicability of this new type of MQMAS experiments to nuclei with larger spins, e.g. ${ }^{27} \mathrm{Al}$ and ${ }^{17} \mathrm{O}$ (both with spin $I=5 / 2$ ). For spin-5/2 nuclei only the $p=0 \rightarrow+3 \rightarrow-1$ pathway signal is refocussed at positive $t_{2}$ values. Thus, for a spin- $5 / 2$ nucleus it is imperative to use sequences like those in Figs. $2 \mathrm{~b}-2 \mathrm{~d}$ that allow acquisition of the $p=0 \rightarrow+3 \rightarrow \cdots \rightarrow-1$ coherence pathway signal. Preliminary theoretical and experimental results in our laboratory show that the sensitivity may be doubled and the efficiency for large quadrupole couplings increased by using modulated-RF mixing on ${ }^{27} \mathrm{Al}$, and similar results have very recently been reported by other groups $(34,35)$. An extended study of improved $p=3 \rightarrow 1$ and $p=5 \rightarrow 1$ quantum coherence mixing schemes for spin-5/2 nuclei will be given elsewhere.

In conclusion, we have demonstrated that it is possible to acquire pure absorption-mode 2D MQMAS spectra by choosing appropriate acquisition schemes in combination with modulated-RF mixing MQMAS experiments. In addition to providing the undistorted second-order lineshape the proposed sequences improve the sensitivity by factors up to $1.3 \mathrm{com}$ pared to the original sequence and 2.75 compared to the single-pulse mixing sequences.

\section{ACKNOWLEDGMENTS}

Financial support from CNRS UPR4212 and Région Centre is acknowledged. T.V. acknowledges financial support from the Carlsberg Foundation. P.J.G. acknowledges support from the National Science Foundation (No. CHE-9807498). The authors thank Profs. A. P. M. Kentgens, L. Frydman, and S. Vega for valuable discussion regarding modulated-RF MQMAS experiments.

\section{REFERENCES}

1. L. Frydman and J. S. Harwood, Isotropic spectra of half-integer quadrupolar spins from bidimensional magic-angle spinning NMR, J. Am. Chem. Soc. 117, 5367 (1995).

2. A. Samoson, E. Lippmaa, and A. Pines, High resolution solid-state N. M. R. averaging of second-order effects by means of a doublerotor, Mol. Phys. 65, 1013 (1988).

3. B. F. Chmelka, K. T. Mueller, A. Pines, J. Stebbins, Y. Wu, and J. W. Zwanziger, Oxygen-17 NMR in solids by dynamic-angle spinning and double rotation, Nature 339, 42 (1989).

4. A. Llor and J. Virlet, Towards high-resolution NMR of more nuclei in solids: Sample spinning with time-dependent spinner axis angle, Chem. Phys. Lett. 152, 248 (1988).

5. P. J. Grandinetti, Dynamic-angle spinning and applications, in "Encyclopedia of Nuclear Magnetic Resonance," (David M. Grant and Robin K. Harris, Eds.), Wiley, Chichester (1995).

6. A. Medek, J. S. Harwood, and L. Frydman, Multiple-quantum magic-angle spinning NMR: A new method for the study of quadrupolar nuclei in solids, J. Am. Chem. Soc. 117, 12779 (1995).

7. G. Wu, D. Rovnyak, and R. G. Griffin, Quantitative multiple quantum magic-angle spinning NMR spectroscopy of quadrupolar nuclei in solids, J. Am. Chem. Soc. 118, 9326 (1996).

8. G. Wu, D. Rovnyak, B. Q. Sun, and R. G. Griffin, High-resolution multiple quantum MAS NMR spectroscopy of half-integer quadrupolar nuclei, Chem. Phys. Lett. 249, 210 (1996).

9. D. Massiot, B. Touzo, D. Trumeau, J. P. Coutures, J. Virlet, P. Florian, and P. J. Grandinetti, Two-dimensional magic-angle spinning isotropic reconstruction sequences for quadrupolar nuclei, Solid-State Nucl. Magn. Reson. 6, 73 (1996).

10. J. P. Amoureux, C. Fernandez, and L. Frydman, Optimized multiple-quantum magic-angle spinning NMR experiments on half-integer quadrupoles, Chem. Phys. Lett. 259, 347 (1996).

11. C. Fernandez and J. P. Amoureux, Triple-quantum MAS-NMR of quadrupolar nuclei, Solid-State Nucl. Magn. Reson. 5, 315 (1996).

12. S. Ding and C. McDowell, Shaped pulse excitation in multi-quantum magic-angle spinning spectroscopy of half-integer quadrupole spin systems, Chem. Phys. Lett. 270, 81 (1997).

13. M. J. Duer and C. Stourton, Further developments in MQMAS NMR spectroscopy for spin-3/2 nuclei, J. Magn. Reson. 124, 189 (1997).

14. S. P. Brown and S. Wimperis, Two-dimensional multiple-quantum MAS NMR of quadrupolar nuclei. Acquisition of the whole echo, J. Magn. Reson. 124, 279 (1997); Two-dimensional multiple-quantum MAS NMR of quadrupolar nuclei: A comparison of methods, J. Magn. Reson. 128, 42 (1997).

15. L. Marinelli, A. Medek, and L. Frydman, Composite pulse excitation schemes for MQMAS NMR of half-integer quadrupolar spins, $J$. Magn. Reson. 132, 88 (1998).

16. S. Ding and C. McDowell, Multiple-quantum MAS NMR spectroscopy of spin-3/2 quadrupolar spin systems using shaped pulses, J. Magn. Reson. 135, 61 (1998).

17. T. Charpentier, C. Fermon, and J. Virlet, Efficient time propagation 
technique for MAS NMR simulation: Application to quadrupolar nuclei, J. Magn. Reson. 132, 181 (1998).

18. T. Mildner, M. E. Smith, and R. Dupree, Rotationally induced triple quantum coherence excitation in MAS NMR spectroscopy of $I=$ 5/2 spins, Chem. Phys. Lett. 301, 389 (1999).

19. S. Vega and Y. Naor, Triple quantum NMR on spin systems with $I=$ $3 / 2$ in solids, J. Chem. Phys. 75, 75 (1981).

20. A. P. M. Kentgens and R. Verhagen, Advantages of double frequency sweeps in static, MAS, and MQMAS NMR of spin $I=3 / 2$ nuclei, Chem. Phys. Lett. 300, 435 (1999).

21. P. K. Madhu, A. Goldbourt, L. Frydman, and S. Vega, Sensitivity enhancement of the MQMAS NMR experiment by fast amplitude modulation of the pulses, Chem. Phys. Lett. 307, 41 (1999).

22. P. K. Madhu, A. Goldbourt, L. Frydman, and S. Vega, Sensitivity enhancement in MQMAS spectroscopy using fast amplitude modulated pulses, Poster M\&T 96, "40th Experimental NMR Conference," Orlando, FL (1999).

23. A. Bax, A. F. Mehlkopf, and J. Smith, Absorption-spectra from phase-modulated spin echoes, J. Magn. Reson. 35, 373 (1979).

24. P. J. Grandinetti, J. H. Baltisberger, A. Llor, Y. K. Lee, U. Werner, M. A. Eastman, and A. Pines, Pure-absorption-mode lineshapes and sensitivity in two-dimensional dynamic-angle spinning NMR, $J$. Magn. Reson. A 103, 72 (1993).

25. R. R. Ernst, G. Bodenhausen, and A. Wokaun, "Principles of Nuclear Magnetic Resonance in One and Two Dimensions," Calendron Press, Oxford (1987).

26. J. P. Amoureux, C. Fernandez, and S. Steuernagel, Z filtering in MQMAS NMR, J. Magn. Reson. A 123, 116 (1996).

27. S. P. Brown, S. J. Heyes, and S. Wimperis, Two-dimensional MAS multiple-quantum NMR of quadrupolar nuclei. Removal of inhomogeneous second-order broadening, J. Magn. Reson. A 119, 280 (1996).

28. D. Massiot, Sensitivity and lineshape improvements of MQ-MAS by rotor-synchronized data acquisition, J. Magn. Reson. A 122, 240 (1996).

29. S. Vega, Fictitious Spin 1/2 operator formalism for multiple quantum NMR, J. Chem. Phys. 68, 5518 (1978).

30. P. K. Madhu, A. Goldbourt, L. Frydman, and S. Vega, manuscript in preparation.

31. C. Dean, T. W. Hambley, and M. R. Snow, Structures of phase IV rubidium nitrate, $\mathrm{RbNO}_{3}$, and phase II caesium nitrate, $\mathrm{CsNO}_{3}$, Acta Crystallogr. C 40, 1512 (1984).

32. J. H. Baltisberger, S. L. Gann, E. W. Wooten, T. H. Chang, K. T. Mueller, and A. Pines, ${ }^{87} \mathrm{Rb}$ dynamic-angle spinning NMR spectroscopy of inorganic rubidium salts, J. Am. Chem. Soc. 114, 7489 (1992).

33. T. Vosegaard, F. H. Larsen, H. J. Jakobsen, P. D. Ellis, and N. C. Nielsen, Sensitivity-enhanced multiple-quantum MAS NMR of halfinteger quadrupolar nuclei, J. Am. Chem. Soc. 119, 9055 (1997).

34. A. Goldbourt, P. K. Madhu, L. Frydman, and S. Vega, Fast RF amplitude modulation in MQMAS NMR spectroscopy, NMR Oral Presentation 219, "41st Rocky Mountain Conference on Analytical Chemistry," Denver, CO (1999); S. Vega, Modulated irradiation fields in solid state NMR, Oral Presentation T18, "1st Alpine Conference on Solid State NMR," Chamonix, France (1999).

35. D. luga and A. P. M. Kentgens, Double frequency sweeps in static, MAS, and MQMAS NMR, Oral Presentation T5, "1st Alpine Conference on Solid State NMR," Chamonix, France (1999). 